\title{
Combined Mobile Ad-hoc and Delay/Disruption-tolerant Routing
}

\author{
Christian Raffelsberger and Hermann Hellwagner \\ Institute of Information Technology, Alpen-Adria Universität Klagenfurt, \\ Klagenfurt, Austria \\ \{craffels, hellwagn\}@itec.aau.at
}

\begin{abstract}
The main assumption of many routing protocols for wireless mobile ad-hoc networks (MANETs) is that end-to-end paths exist in the network. In practice, situations exist where networks get partitioned and traditional ad-hoc routing fails to interconnect different partitions. Delay/disruption-tolerant networking (DTN) has been designed to cope with such partitioned networks. However, DTN routing algorithms mainly address sparse networks and hence often use packet replication which may overload the network. This work presents a routing approach that combines MANET and DTN routing to provide efficient routing in diverse networks. In particular, it uses DTN mechanisms such as packet buffering and opportunistic forwarding on top of traditional ad-hoc end-to-end routing. The combined routing approach can be used in well-connected networks as well as in intermittently connected networks that are prone to disruptions. Evaluation results show that our combined approach can compete with existing MANET and DTN routing approaches across networks with diverse connectivity characteristics.
\end{abstract}

Keywords: mobile ad-hoc networks, disruption-tolerant networks, routing, simulation

\section{Introduction}

The majority of state-of-the-art routing protocols [1] for wireless mobile adhoc networks (MANETs) assume the existence of an end-to-end path between source and destination pairs. These protocols fail to deliver packets if such an end-to-end-path does not exist. However, in real application scenarios, ad-hoc networks may not be fully connected since disruptions cause the network to get partitioned. In practice, many ad-hoc networks will provide well-connected regions but still suffer from partitioning which prevents end-to-end communication between a subset of the nodes. A reason for such disruptions are link failures caused by obstacles or the mobility of nodes. Diverse connectivity characteristics impose challenges on the communication network, especially on the routing protocol. Hence, there is a need for hybrid routing protocols that exploit multihop paths to efficiently route packets in well-connected parts of the networks and permit inter-partition communication by storing packets that cannot be 
routed instantly. One example for networks that are prone to partitioning are hastily formed ad-hoc networks for emergency response operations. These networks may be diverse in terms of connectivity and networking equipment. The connectivity may range from well-connected networks, where nearly all nodes are interconnected, to sparse networks, where most nodes are disconnected. In between these two extremes, the network may also be intermittently connected and provide several 'islands of connectivity'. For instance in disaster response scenarios, which are a promising application domain for mobile ad hoc networks, members from the same search and rescue team may be interconnected as they tend to work near each other. However, there may be no end-to-end paths available between different teams or the incident command center and teams that are spread on the disaster site. MANET protocols that try to find end-to-end paths will not work satisfactorily in such emergency response networks that provide diverse connectivity characteristics [6].

Routing algorithms for Delay- or Disruption-Tolerant Networking (DTN) [8] do not assume the existence of end-to-end paths but allow nodes to store messages until they can be forwarded to another node or delivered to the final destination. This mechanism is called store-and-forward or store-carry-forward routing and increases robustness in the presence of network disruptions. However, many DTN routing algorithms use packet replication to improve delivery probability and delivery delay. Whereas this mechanism is beneficial in sparse networks that provide few contact opportunities, it may dramatically decrease performance in dense networks, since it introduces high overheads in terms of transmission bandwidth and storage.

The contributions of this paper are as follows. We introduce a combined MANET/DTN routing approach called CoMANDR that extends end-to-end MANET routing with mechanisms from DTN routing. CoMANDR is designed to cover a broad range of connectivity characteristics, from intermittently connected to well-connected networks. The combined routing approach makes no assumption about the existence of end-to-end paths. It can deliver packets instantly if end-to-end paths exist or select custodian nodes opportunistically to bridge islands of connectivity. We evaluate our approach in several scenarios and compare it with other state-of-the-art routing approaches from the MANET and the DTN domain.

The remainder of the paper is structured as follows. Section 2 introduces the routing protocols that are used in the evaluation. Section 3 describes the design of CoMANDR. Section 4 presents the evaluation setup including a scenario description and the used metrics. The simulation results are discussed in Section 5. Finally, Section 6 concludes the paper and discusses possible future work.

\section{Related Work}

This section briefly describes the protocols that are used in the evaluation. PROPHET [5] is a flooding-based DTN routing protocol that uses the so called delivery predictability metric to decide to which nodes a message should be 
forwarded. The delivery predictability is a measure of how likely it is that a node can deliver a message to its destination. It is based on the assumption that nodes that have met frequently in the past, are also likely to meet again in the future. Whenever two nodes meet, they exchange and update their delivery predictability values and exchange all messages for which the other node has a higher delivery probability. For this evaluation, CoMANDR uses PROPHET's delivery predictability metric in its utility calculation function (see Section 3.2 for details). CoMANDR is dependent on a MANET routing protocol that finds end-to-end paths in the network. We did not choose a specific MANET routing protocol for the evaluation. Instead, we use a generic link state protocol, referred to as MANET, that finds the shortest end-to-end paths in the network and is capable of routing packets in connected parts of the network. The MANET routing protocol implementation supports to limit the maximum length of end-to-end paths that are reported. By limiting the path length it is possible to simulate imperfections of MANET routing protocols in real networks. Without this limitation, the packet delivery ratio of MANET represents the upper bound for all protocols that rely on end-to-end paths. The same MANET routing protocol is used in CoMANDR to build the routing tables and route packets if a path is available. Some recent approaches that combine MANET and DTN routing have added packet buffering to a MANET routing protocol [2][7]. These approaches buffer packets instead of dropping them if no end-to-end path is available. We added packet buffering to the optimal MANET routing protocol to simulate this kind of approach. The resulting protocol is called MANET store-and-forward (MANET-SaF) and is one example for hybrid MANET/DTN routing. Additionally, the evaluation includes the Epidemic routing protocol [9]. Epidemic routing floods the whole network. In particular, whenever two nodes meet, Epidemic routing exchanges all messages that the other node has not already buffered. If transmission bandwidth and buffers are unlimited, Epidemic would utilize all available routes and optimize delivery delay and packet delivery ratio. Hence, Epidemic sets the upper bound for the performance of any routing algorithm. However, Epidemic's high resource usage negatively affects its performance in resource-constraint environments.

\section{Combined MANET/DTN Routing}

Combined MANET/DTN Routing (CoMANDR) works like a traditional routing protocol for MANETs when end-to-end paths are available. It uses the routing table that is calculated by the MANET protocol to route packets that can be reached instantly over a multi-hop end-to-end path. Thus, CoMANDR will exactly work like the underlying MANET routing protocol if the network is fully connected. To cope with disruptions, CoMANDR utilizes two mechanisms from delay/disruption-tolerant networking: packet buffering and utility-based forwarding. If the routing table contains no valid entry for a packet's destination, CoMANDR buffers the packet instead of discarding it. The rationale behind this behavior is that a buffered packet may be sent later when a route becomes avail- 
able (i.e., sender and receiver are in the same connected component). There may be situations where an end-to-end path between sender and receiver will never be available. To handle such situations, CoMANDR may also forward packets to nodes that are assumed to be closer to the destination. The decision to which node a buffered packet should be forwarded is based on a utility function. One interesting aspect is that the utility function can re-use information that is collected by the MANET routing protocol (e.g., information from the routing table or link-state announcements). However, it is also possible to collect additional information to calculate utility values for other nodes in the network. The utility values are used to determine an alternative path if no end-to-end path has been found. Nodes with higher utility values are more likely to deliver packets to the destination. In general, CoMANDR first tries to send a packet via available MANET routes. If this is not possible, the packet is sent to the neighbor with the highest utility value for that packet. While this procedure is repeated, the packet is sent hop-by-hop towards the destination. The following pseudo code describes the basic algorithm to combine MANET and DTN routing:

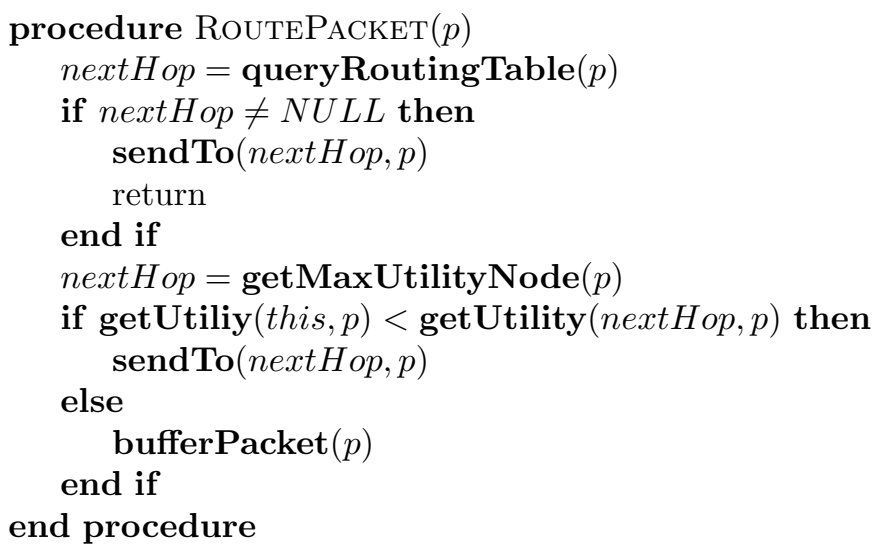

\subsection{Packet Buffering}

In order to provide store-carry-forward routing, packets need to be buffered when no end-to-end path is available. Additionally, it is checked if a routing table entry is valid. The packet delivery ratio can be increased if the validity of routes is checked and packets are buffered in case of stale routes [7]. An entry is invalid if its next hop entry is currently not available (i.e., there is no wireless link to the one hop neighbor that is advertised by the entry). Such stale route entries may be an effect of link outages caused by the mobility of nodes or by obstacles and MANET routing protocols need some time to detect and handle such events. To identify if a next hop is available, MANET routing control traffic can be monitored [7]. Additionally, information from other layers may be used. For instance, information about the status of links that are provided by the underlying link layer protocol.

Apart from deciding when to buffer a packet, it is also important to decide when a buffered packet can be sent. In case of temporary link outages, packets 
may be sent as soon as the link is available again or a proactive MANET routing protocol has provided an alternative path that includes a valid next hop. However, there may be cases where no end-to-end path will be found since the destination of a buffered packet is in another partition. In these cases it is beneficial to forward the packet to a node that is more likely to deliver the message. This mechanism is called utility-based forwarding and is described in the next section. It is important to note that the evaluated version of CoMANDR only forwards a single copy of every packet since every node deletes a packet that it has forwarded to another node. This saves transmission bandwidth and storage but may negatively affect routing performance in sparse networks.

\subsection{Utility-based Forwarding}

The utility of a node describes the node's fitness to deliver a packet towards its destination. In general, a node will hand over a packet to another node if the other node has a higher utility value. The utility may be dependent or independent of the destination [8]. A destination-independent utility function is based on characteristics of the potential custodian node, such as its resources or mobility. On the other hand, destination-dependent utility functions are based on characteristics concerning the destination, such as how often a node has met the destination or if a node and the destination belong to the same social group.

The combined use of a utility table and a MANET routing table allows nodes to route packets in both connected and disrupted networks. The MANET routing table represents some sort of spatial information (i.e., which nodes are currently in the vicinity of a node). Combining routing table information with utility functions that contain historic data (e.g., information about previous states of the routing table), effectively calculates spatio-temporal clusters of nodes. This information allows a node to determine to which other node a packet should be sent, when there is currently no end-to-end path to the destination available.

The performance of a utility function is influenced by the characteristics of the scenario. Hence, it is important to choose a utility function or a combination of functions that fits the specifics of the intended application scenario. We have chosen a utility function that uses routing table entries to calculate meeting probabilities based on the well-known PROPHET routing algorithm [5] for DTNs. Although we performed some experiments with different parameters for the utility calculation, to empirically determine parameters that suit the scenarios, it is important to note that the purpose of this work is not to find an optimal utility function. Instead, this work intends to show that a combination of MANET routing and DTN routing (i.e., applying mechanisms such as packet buffering and utility-based forwarding on top of a MANET routing protocol) is beneficial in some scenarios.

To limit the calculation efforts for the utility function and the amount of data to be exchanged, every node should limit the number of nodes it keeps in its utility table. One possibility is that every node only keeps the $n$ highest entries in its utility table. Another possibility is to remove nodes if their utility value drops under a certain minimum threshold. The second option was used for 
the evaluation (i.e., nodes are removed from a utility table if their utility value drops below 0.2 ). If nodes are not in the utility table of another node, they will not be used as custodian nodes. This prevents nodes from forwarding packets to custodians that only offer a low chance to deliver the packet to its destination. Otherwise, a lot of transmissions would be performed without significantly increasing the delivery probability.

CoMANDR uses a modified version of the PROPHET meeting probability calculation function to calculate the utility of a node. In contrast to the PROPHET protocol, that only considers when two nodes directly meet (i.e., there is a direct link between the nodes), CoMANDR also considers multi-hop information from the routing table. When a node $i$ has a routing table entry for another node $j$ (with a distance less than infinite), CoMANDR considers node $i$ and $j$ to be in contact. This allows nodes to exploit multi-hop paths to determine contacts with other nodes.

As the MANET routing protocol regularly updates the routing table entries, the meeting probabilities and thus the utility values for other nodes need to be updated as well. To be precise, every node $i$ manages one utility value for every node $j$ that it knows. The set of known nodes includes all destinations for which a routing entry exists or has existed previously (i.e., disconnected nodes that are still kept in the cluster). So if a route to node $j$ is known, node $i$ will update the utility value for node $j$ (denoted as $U_{i j}$ ) using PRoPHET's probability update function:

$$
U_{i j}=U_{i j}+\left(1-U_{i j}\right) * \alpha
$$

On the other hand, for a node $k$ that is not in the routing table but has a utility value, the utility value $U_{i k}$ is reduced:

$$
U_{i k}=U_{i k} * \gamma
$$

The parameter $\alpha$ determines how fast the utility converges to 1 if there is a contact between two nodes, whereas $\gamma$ determines how fast it converges to 0 if there is no contact. Both parameters need to be in the range between 0 and 1 .

Every node needs to regularly broadcast all calculated utilities (the utility table) to its direct neighbors. When a node receives the utility table of another node, it can use this information to update its own utility table. If a node $i$ is in contact with node $j$ that has a utility value for node $k$, node $i$ can transitively update its utility value for node $k$. For instance using PROPHET's transitive update function:

$$
U_{i k}=\max \left(U_{i k \text { old }}, U_{i j} * U_{j k} * \beta\right)
$$

$\beta$ is used to control the impact of transitivity. It is worth noting that the transitive update function is general and not tied to the use of PROPHET's meeting probability.

\section{Evaluation Setup and Scenarios}

The overall goal of the evaluation is to show that CoMANDR performs well in a broad range of connectivity settings. The Opportunistic Network Environ- 
ment (ONE) simulator [4] is used to evaluate all protocols. The ONE is mainly intended to evaluate DTN routing protocols. It focuses on the network layer and does not implement physical characteristics of the transmission. Although this imposes a lack of realism, we believe that it is still possible to make a fair comparison between MANET, DTN and our hybrid MANET/DTN approach.

We needed to implement multi-hop MANET routing within the ONE. In particular, we implemented a link state protocol that uses Dijkstra's shortest path algorithm to calculate the shortest paths in the network. This link state MANET routing protocol is also used by CoMANDR to route packets in the connected parts of the network. Hop count is used as route metric, as the ONE does not provide any information about the quality of links. All nodes have the same view on the network. Thus, the implemented MANET routing protocol is optimal. In reality, routing protocols have to cope with imperfect information about the network (e.g., information about links is missing or wrong). Hence, routing protocols have problems to find end-to-end paths in mobile scenarios. In particular, paths that comprise many hops may not be found. Additionally, the throughput of end-to-end paths drastically decreases with the hop count [3]. Thus, we restrict the maximum length of paths that are reported by the MANET routing algorithm to simulate these problems. If not denoted otherwise, the routing table only includes routes with a maximum end-to-end path length of five hops for all experiments. This restricts the maximum path length that MANET can exploit to five. All other protocols may still exploit longer paths as they do not only use end-to-end paths but also store-and-forward routing to deliver packets.

\subsection{Scenarios}

All protocols are evaluated in several scenarios that offer different connectivity characteristics. In a first set of experiments we varied the transmission range and simulation area size to get a diverse set of networking scenarios, ranging from well-connected to sparse networks. We calculated the connectivity degree for all scenarios (see 4.2) and selected three scenarios offering different levels of connectivity. In particular, we selected three scenarios that use the same transmission range of $100 \mathrm{~m}$ but have a different simulation area size. The selected scenarios include a well-connected scenario, a sparse scenario and an intermittently connected scenario that lies between the other two.

The mobility model that is used in all scenarios is the random walk model as implemented in the ONE. In particular, a node selects its next destination by randomly selecting a direction, speed and distance, after waiting for a random pause time. Since the maximum distance between two consecutive waypoints is limited, nodes moving according to this model tend to stay close to each other for a longer time, compared to the random waypoint model. It is important to note that random mobility rather puts PROPHET and CoMANDR at a disadvantage because both protocols assume that the future encounter of nodes is predictable. However, we argue that the low movement speed of nodes (i.e., the max speed is $2 \mathrm{~m} / \mathrm{s}$ ) and the fact that consecutive waypoints are close to each other, mitigate 
Table 1. Simulation parameters

\begin{tabular}{|c|c|}
\hline \multicolumn{2}{|c|}{ Mobility model } \\
\hline No. nodes & 100 \\
\hline Model & Random Walk \\
\hline Movement speed & 1 to $2 \mathrm{~m} / \mathrm{s}$ \\
\hline Pause time & 0 to $60 \mathrm{~s}$ \\
\hline Distance (min,max) & 0 to $50 \mathrm{~m}$ \\
\hline \multicolumn{2}{|c|}{ Wireless settings } \\
\hline Transmission range & $100 \mathrm{~m}$ \\
\hline Transmission rate & $4 \mathrm{Mbps}$ \\
\hline \multicolumn{2}{|c|}{ Traffic model } \\
\hline \multicolumn{2}{|c|}{ Packet creation interval 500 to $2500 \mathrm{~s}$} \\
\hline Packet creation rate & 1 msg every $30 \mathrm{~s}$ (per node) \\
\hline Packet size & $100 \mathrm{kB}$ \\
\hline Packet buffer size & $700 \mathrm{MB}$ (per node) \\
\hline \multicolumn{2}{|c|}{ Parameter for PROPHET routing/CoMANDR } \\
\hline$P_{\text {init }}(=\alpha)$ & 0.9 \\
\hline$\beta$ & 0.7 \\
\hline$\gamma$ & 0.995 \\
\hline
\end{tabular}

the effects of random mobility to some extent. For instance, two nodes that have met recently are also more likely to meet each other again than two nodes that are far away from each other. Moreover, it has been shown that PROPHET is still able to perform reasonably well in scenarios with random mobility [5].

The total simulation time is $4500 \mathrm{~s}$ and all experiments are repeated 23 times using different seeds for the mobility model and the traffic generator. All scenarios include 100 nodes. Traffic is generated by creating a new packet with random source and destination every $0.3 \mathrm{~s}$. Hence, a node generates a new packet every $30 \mathrm{~s}$ on average. No traffic is generated after $2500 \mathrm{~s}$ to allow the routing protocols to deliver buffered packets before the simulation ends. All packets have an infinite time to live. Important simulation parameters are listed in Table 1.

\subsection{Metrics}

The first metric that is used to evaluate the routing approaches is the packet delivery ratio (PDR). It shows the ratio between successfully received packets at the destination and the number of created packets. The hop count shows how many nodes a packet has passed from source to destination. The transmission cost metric denotes the ratio between transmitted packets and successfully received packets. For single-copy schemes such as MANET routing and CoMANDR, the transmission cost is proportional to the average hop count of all successfully received messages. For the other schemes, the transmission cost is mainly influenced by the number of message replicas. The latency represents the time that is 
needed to transfer a packet from the source to the destination. Latency includes the buffering time and the transmission time for all nodes along the path.

A metric that is often used for evaluating mobile ad-hoc routing protocols is the routing control overhead (i.e., the traffic overhead for finding end-to-end routes). However, different MANET routing protocols greatly vary in the amount of control overhead they introduce [10]. As this study only includes a generic MANET protocol, it is not feasible to directly measure control overhead. As CoMANDR and MANET-SaF are extensions of the generic MANET protocol, the overhead for these three protocols is comparable. It is also fair to assume that the control overhead of the underlying MANET routing protocol is significantly lower than the data overhead introduced by the multi-copy schemes that are evaluated in this paper. Hence, we argue that not taking control overhead into account should not hinder a fair comparison of the evaluated protocols.

Three additional metrics are used to characterize the network connectivity of the simulation scenarios. The connectivity degree $C D$ is the probability that two randomly selected nodes are in the same connected component at a given point in time (i.e., an end-to-end path between the two nodes exists). A connectivity degree of 1 denotes a fully connected network, whereas 0 denotes a network were all nodes are isolated. The connectivity degree at a given point in time $t$ is calculated as follows:

$$
C D_{t}=\sum_{P_{i} \in P_{t}} \frac{\left|P_{i}\right|}{|N|} * \frac{\left|P_{i}\right|-1}{|N|-1},
$$

where $N$ denotes the set of all nodes in the network and $P_{t}$ denotes the set of partitions that comprise the network at a given time $t .\left|P_{i}\right|$ denotes the number of nodes in one particular partition and $|N|$ the total number of nodes in the network. As the connectivity degree changes over time, the average connectivity degree for the duration of the simulation has to be calculated as follows:

$$
C D=\frac{1}{T} * \sum_{t}^{T} C D_{t},
$$

where $T$ denotes the number of samples that have been taken and $C D_{t}$ the connectivity degree for one sample. For the scenarios in this paper, $C D$ denotes the mean value of 4500 samples (i.e., one sample per second). Another metric that describes the connectivity of a network is the largest connected component (LCC). The LCC denotes the number of nodes that are located in the largest partition. The third metric used to characterize the scenario in terms of connectivity is the number of partitions with at least two nodes. Hence, the number of partitions does not include isolated nodes. Table 2 lists the connectivity characteristics for the evaluation scenarios.

\section{Results}

This section includes the evaluation results for CoMANDR, Epidemic, PROPHET, MANET and MANET-SaF. Unless otherwise stated, figures show mean values of all simulation runs and error-bars denote the $95 \%$ confidence interval. 
Table 2. Scenario characteristics in terms of network connectivity.

\begin{tabular}{r|r|r|r}
\hline $\begin{array}{r}\text { Size of area } \\
\text { (in } \mathrm{m} \mathrm{x} \mathrm{m)}\end{array}$ & $\begin{array}{r}\text { Avg. connectivity } \\
\text { degree } C D\end{array}$ & $\begin{array}{c}\text { Largest connected } \\
\text { component (avg) }\end{array}$ & $\begin{array}{r}\text { Avg. no of } \\
\text { partitions }\end{array}$ \\
\hline $700 \times 700$ & 0.882 & 92.886 & 1.915 \\
\hline $800 \times 800$ & 0.634 & 74.95 & 3.853 \\
\hline $1000 \times 1000$ & 0.157 & 30.276 & 17.982 \\
\hline
\end{tabular}

The packet delivery ratio for all evaluated protocols in the three scenarios is shown in Figure 1a. Traditional end-to-end MANET routing is clearly outperformed by the other protocols and achieves the lowest PDR in all scenarios. Epidemic routing can deliver most packets in all scenarios. This is due to the fact that the link bandwidth is very high and nodes can store all packets in their buffers, which is the ideal case for Epidemic. No packets are dropped because of full buffers which maximizes Epidemic's performance. PROPHET can achieve a similar PDR in well-connected and intermittently connected scenarios. The performance results of CoMANDR and MANET-SaF are comparable in the well-connected scenario. The reason for this is that source and destination are very likely to be in the same connected component at some point in time and the packets can be delivered via an end-to-end path. Hence, MANET-SaF works similarly to CoMANDR in this scenario and both protocols achieve nearly the same PDR. However, CoMANDR outperforms MANET-SaF in the other two scenarios. In the sparse scenario, CoMANDR could deliver nearly $50 \%$ more packets than MANET-SaF. This performance gain is achieved by the utility-based forwarding scheme of CoMANDR that forwards packets towards the destination. Thus, CoMANDR can deliver packets to destinations that are never in the same connected component as the source, which improves its performance compared to MANET-SaF.

The protocols are diverse in terms of transmission cost as shown in Figure 1b. Due to its aggressive replication scheme, Epidemic nearly performs 100 packet transmissions to deliver one packet. Although PROPHET can reduce this number by not forwarding packets to neighbors that have a lower delivery predictability, it still replicates packets extensively. MANET produces the lowest transmission cost as it only delivers packets via the shortest available end-to-end path. As the path has to be available instantly, it drops packets if it fails to find such an end-to-end path. MANET-SaF has a higher transmission cost than MANET as buffering packets instead of dropping them allows it to deliver more packets, especially via longer paths. CoMANDR has a higher transmission cost if the connectivity is low. However, compared to the multicopy schemes Epidemic and PROPHET, its transmission cost is still very low. Thus, CoMANDR offers the best trade-off between packet delivery ratio and transmission cost among all protocols. We believe that this is a very important feature of CoMANDR as resources are often scarce in mobile networks. Reducing the number of transmissions and hence reducing the wireless channel utilization 


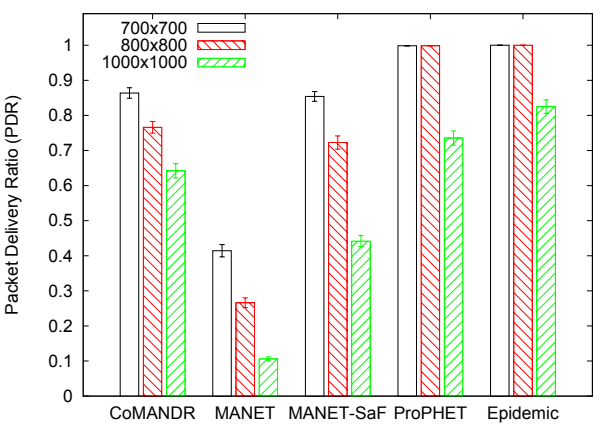

(a) PDR

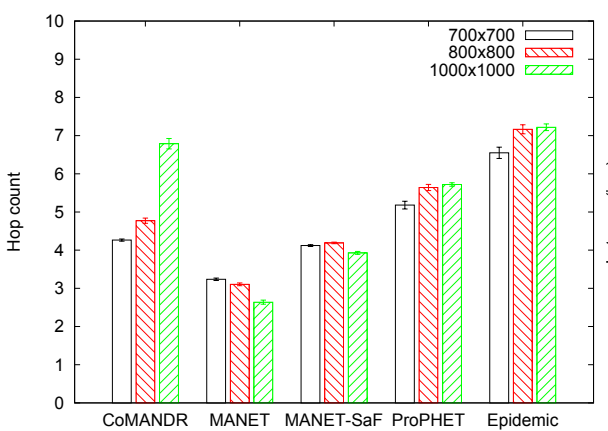

(c) Hop count

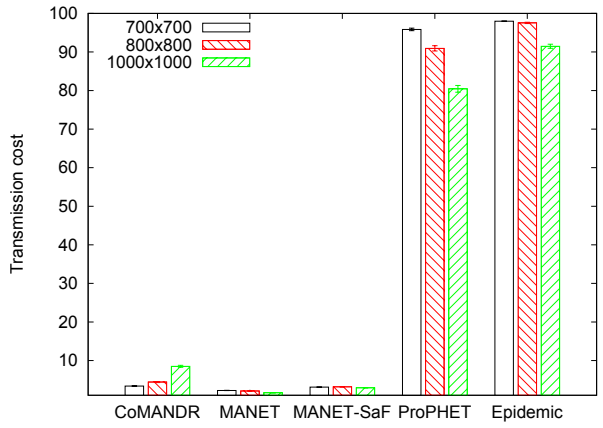

(b) Transmission cost

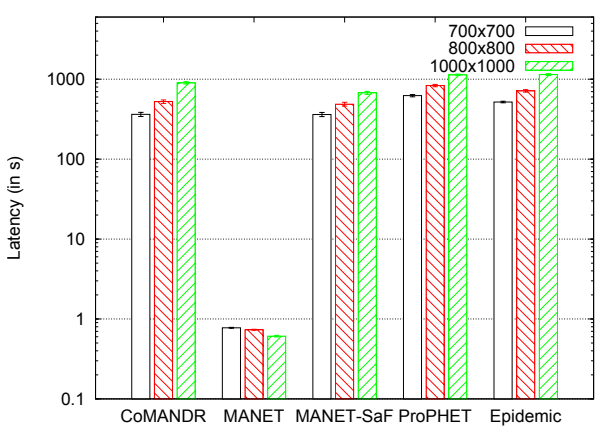

(d) Latency

Fig. 1. Performance comparison for scenarios with different connectivity.

and battery consumption, while still providing a good packet delivery ratio, is an important issue in many scenarios.

The hop count is shown in Figure 1c. In general it can be said that, for the same scenario, the hop count is correlated with the packet delivery ratio. In particular, the protocols that achieve a higher packet delivery ratio achieve this mainly by utilizing longer paths which increases the average hop count. Since MANET only delivers packets via end-to-end paths, its hop count is limited by the fact that end-to-end paths do not comprise many hops, especially in the sparse scenario. Additionally, as long end-to-end paths have been removed from the routing table to simulate imperfections of MANET protocols in real networks, the maximum hop count is limited. We also performed some experiments with a higher hop limitation for end-to-end paths. With higher hop limitations, MANET also utilizes longer paths and the average hop count is higher. Due to space constraints, we cannot present detailed results about hop count for these experiments. As mentioned before, MANET-SaF can deliver more packets via longer paths as it stores packets if no end-to-end path is available, or the end-toend path breaks while the packet is on its way to the destination. Similarly, the multi-copy schemes Epidemic and PROPHET have a higher hop count as they 
are able to deliver more packets via long paths. The hop count of CoMANDR is similar to the one of MANET-SaF for the well-connected and intermittently connected scenarios. In the sparse scenario, CoMANDR's utility-based forwarding technique finds more paths but also needs more hops. However, as only one message copy is passed in the network, this does not cause a high transmission cost.

Latency is shown in Figure 1d. Since MANET only uses instantly available end-to-end paths, it has the lowest latency. However, at the cost of a low PDR. The other protocols have a significantly higher latency due to packet buffering. Similar to the hop count, the latency is correlated with the PDR.

We also performed experiments with a varying hop count limit for the endto-end paths. As mentioned before, MANET routing protocols often fail to find multi-hop paths including many hops, especially in mobile scenarios. For the previous experiments, we limited the maximum path length to five which is a rather conservative estimation and limits the performance of MANET and protocols depending on it (i.e., CoMANDR and MANET-SaF that use MANET to route packets in the connected parts of the network). Figure 2 shows how the PDR is affected by the length limitation of end-to-end paths. An interesting finding is that the store-and-forward mechanism of MANET-SaF and CoMANDR is a good means to increase the PDR, when the MANET protocol does not find longer multi-hop paths. For instance, in the intermittently connected scenario (see Fig. 2b), CoMANDR with a relatively strict maximum end-to-end path length of four achieves a higher PDR than MANET with practically no restriction (i.e., hop limit 20). Even in the well-connected scenario, idealistic MANET routing has a lower PDR than CoMANDR and MANET-SaF for hop limits greater than five (see Fig. 2a). This is an indication that CoMANDR may also perform better than traditional MANET protocols in well-connected but quickly changing networks, where traditional MANET protocols fail to find end-to-end paths because of the mobility of nodes.

We also performed experiments to assess the performance of CoMANDR using different values for $\alpha, \beta$ and $\gamma$. Due to space constraints we cannot present the results in detail. However, results show that the aging factor $\gamma$ has a higher impact on routing performance than $\alpha$ and $\beta$. Especially in scenarios with low connectivity, $\gamma$ should be set to a high value as this increases the PDR, without increasing the transmission cost significantly. The values listed in Table 1 offered the best performance over all scenarios.

In the given scenarios, the packet delivery ratio of CoMANDR is always better than or equal to the delivery ratio of MANET and MANET-SaF routing. This shows that the mechanisms applied by CoMANDR on top of MANET routing, namely packet buffering and utility-based forwarding, are beneficial. In contrast to MANET routing, CoMANDR achieves packet delivery ratios that are comparable to state-of-the-art DTN routing algorithms in the intermittently and low connected scenarios. It is worth noting that sufficiently large buffers were provided in all scenarios. This is very beneficial for Epidemic and PROPHET since the packet delivery ratio is not negatively affected by packet drops caused 


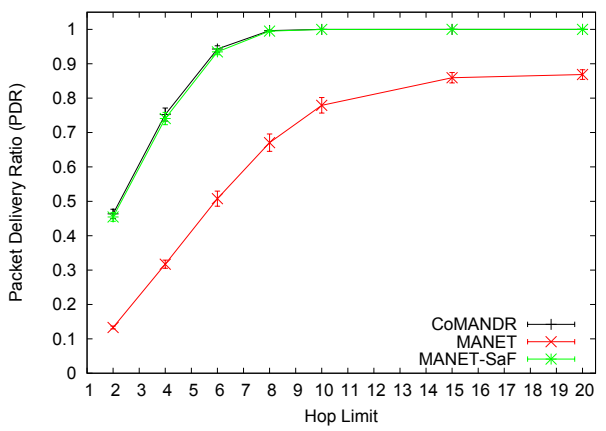

(a) PDR for $700 \times 700 \mathrm{~m}$

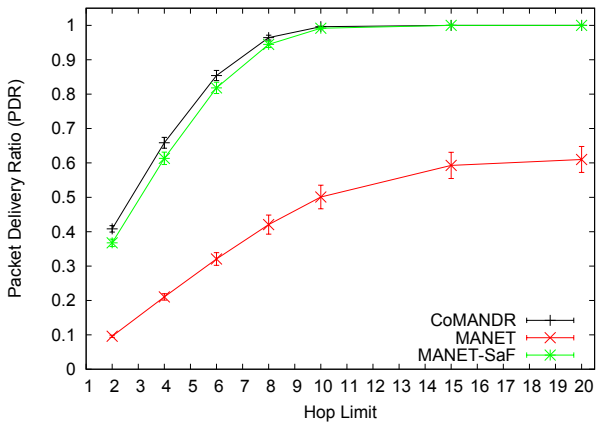

(b) PDR for $800 \times 800 \mathrm{~m}$

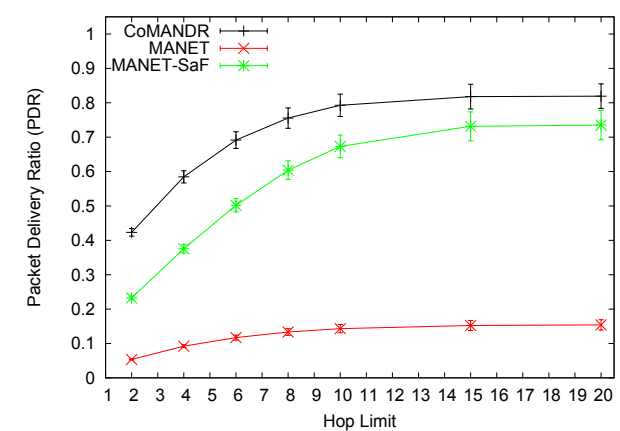

(c) PDR for $1000 \times 1000 \mathrm{~m}$

Fig. 2. Packet delivery ratio for different end-to-end path hop limits.

by full buffers. On the other hand, CoMANDR is much more efficient. Thus, the performance of CoMANDR will obviously be less affected by limited resources. This shows that CoMANDR is well suited for a broad range of networks.

\section{Conclusion}

CoMANDR combines MANET and DTN routing in order to ensure good performance across a broad range of networks. In well-connected networks, it works similar to traditional MANET routing. Additionally, it uses mechanisms to store and opportunistically forward packets to custodian nodes if no end-to-end path exists. Evaluation results show that our approach can compete with or outperform other state-of-the-art routing protocols both from the MANET and DTN domain. One important advantage of CoMANDR is that it offers a good trade-off between packet delivery ratio and transmission cost. As the intended application scenarios of CoMANDR include networks consisting of resource-constrained mobile devices, using resources efficiently is a very important feature of the protocol. 
For this evaluation CoMANDR was implemented as single-copy scheme. However, it would be interesting to assess its performance if packet replication were used. This should improve CoMANDR's performance in very sparse networks. However, as the intended application domain of CoMANDR are diverse networks, it is important to design a replication scheme that does not introduce too high overheads concerning storage and bandwidth which would decrease performance in well-connected parts of the network and waste possibly scarce resources such as transmission bandwidth, battery or storage. Other topics for future work are to look into additional utility functions and evaluate CoMANDR in realistic scenarios such as emergency response operations, that are an interesting application domain for this kind of routing approach.

Acknowledgments The research leading to these results has received funding from the European Union 7th Framework Programme (FP7/2007-2013) under grant agreement no 261817, the BRIDGE project, and was partly performed in the Lakeside Labs research cluster at Alpen-Adria-Universität Klagenfurt.

\section{References}

1. Boukerche, A., Turgut, B., Aydin, N., Ahmad, M., Bölöni, L., Turgut, D.: Routing protocols in ad hoc networks: A survey. Computer Networks 55(13), 3032-3080 (Sep 2011)

2. Delosieres, L., Nadjm-Tehrani, S.: Batman store-and-forward: the best of the two worlds. In: Proc. Int. Conf. Pervasive Computing and Communications Workshops (PerCom Workshops '12). pp. 721 -727. IEEE (2012)

3. Johnson, D., Hancke, G.: Comparison of two routing metrics in OLSR on a grid based mesh network. Ad Hoc Networks 7(2), 374-387 (Mar 2009)

4. Keränen, A., Ott, J., Kärkkäinen, T.: The ONE simulator for DTN protocol evaluation. In: Proc. 2nd Int. Conf. Simulation Tools and Techniques (SIMUTools '09). pp. 55:1-55:10. ICST (2009)

5. Lindgren, A., Doria, A., Schelén, O.: Probabilistic routing in intermittently connected networks. SIGMOBILE Mob. Comput. Commun. Rev. 7, 19-20 (July 2003)

6. Raffelsberger, C., Hellwagner, H.: Evaluation of MANET routing protocols in a realistic emergency response scenario. In: Proc. Int. Workshop on Intelligent Solutions in Embedded Systems (WISES '12). pp. 88 - 92. IEEE (2012)

7. Raffelsberger, C., Hellwagner, H.: A hybrid MANET-DTN routing scheme for emergency response scenarios. In: Proc. Int. Conf. Pervasive Computing and Communications Workshops (PerCom Workshops '13). pp. 505 - 510. IEEE (2013)

8. Spyropoulos, T., Rais, R.N., Turletti, T., Obraczka, K., Vasilakos, A.: Routing for disruption tolerant networks: Taxonomy and design. Wireless Networks 16(8), 2349-2370 (Nov 2010)

9. Vahdat, A., Becker, D.: Epidemic routing for partially-connected ad hoc networks. Tech. Rep. CS-2000-06, Duke University (Jul 2000)

10. Viennot, L., Jacquet, P., Clausen, T.H.: Analyzing control traffic overhead versus mobility and data traffic activity in mobile ad-hoc network protocols. Wireless Networks 10(4), 447-455 (Jul 2004) 